\title{
Electrostatic Analogy for Surfactant Assemblies
}

\author{
David Wu, ${ }^{\dagger}$ David Chandler, ${ }^{*}$ and Berend Smit ${ }^{\ddagger}$
}

Department of Chemistry, University of California, Berkeley, Berkeley, California 94720

(Received: November 13, 1991)

\begin{abstract}
We develop a concept of frustrating charges to create a theory of self-assembly. In particular, we note that the constraints of stoichiometry frustrate ordinary phase equilibria and lead to self-assembly of systems such as oil-water-surfactant mixtures. Further we note that at long wavelengths, the constraints of stoichiometry are isomorphic to the constraints of charge neutrality in a specific electrostatic analogy. We expand upon this analogy, first noted by Stillinger, and show that it can be used to derive useful analytical estimates. In addition we use the analogy to create a new model for frustrated systems, and we present Monte Carlo results for this charge frustrated Ising system that exhibits varied behaviors of self-assembly. The Monte Carlo calculations are made possible through the development of an algorithm which permits cluster moves.
\end{abstract}

\section{Introduction}

The physics of self-assembly in surfactant-oil-water mixtures ${ }^{1}$ is intimately connected with that of frustration. ${ }^{2}$ Recent computer simulations of Smit and co-workers illustrate this point. ${ }^{3,4}$ In particular, consider a system which separates into one phase rich in component $A$ and another phase rich in component $B$. The phase equilibrium can be "frustrated" by constraining an excess of species $A$ to reside in the rich $B$ phase (or by constraining an excess of $B$ to be in the A phase). The frustration can be accomplished physically by forming $\mathrm{A}_{n} \mathrm{~B}_{m}$ molecules from a fraction of the A and B particles. This formation is, in fact, what Smit and co-workers do with a small percentage of simple fluid particles. The bound $\mathbf{A}_{n} \mathbf{B}_{m}$ species are the surfactants in that system. After enough such surfactants are introduced, the interface becomes saturated, and the remaining surfactants are forced into one of the two bulk phases. The stoichiometric constraint therefore forces, for example, excess B particles into the rich A phase. The resulting system organizes into assemblies-micelles, bilayers, and the like.

In this paper, we focus attention on the role of stoichiometric constraints. We argue that at long distance scales, the role of those constraints are isomorphic with that of electroneutrality in a system of free charged particles. Specifically, one may associate a charge with some fraction of $\mathbf{A}$ particles, and an opposite sign charge with a similar fraction of B particles. It is clear that the Coulombic interactions in such a system will oppose and frustrate a system that would otherwise simply phase separate. The connection between charge-charge interactions and the constraints of stoichiometry are not, however, obvious. Nevertheless, the connection can be made, as Stillinger has noted before us, ${ }^{5}$ provided care is taken in identifying the magnitudes of the charges.

The motivation and specification of the electrostatic analogy is given in section 2. The analogy is then used in section 3 to develop a qualitative scaling theory of self-assembly. This development provides some feeling for how models based on this picture will work. Additional insight may be gleaned from lattice simulations - though our ultimate reason for considering this class of models is to devise analytical off-lattice theories. In section 4, a frustrated Ising model based on the electrostatic analogy is introduced and studied in two dimensions by Monte Carlo simulation. It is shown that the model does indeed exhibit the phenomena of self-assembly. The implication, therefore, is that the long-wavelength manifestation of stoichiometric constraints and its competition with phase equilibria provide a sufficient mechanism for self-assembly. Further microscopic detail is not intrinsic to the phenomena. We conclude in section 5 with a brief discussion.

\footnotetext{
${ }^{\dagger}$ Present address: Cavendish Laboratory, Madingley Road, Cambridge CB3 OHE, UK.

IVisiting the University of California from Koninklijke/Shell-Laboratorium, Amsterdam (Shell Research B.V.), P.O. Box 3003, 1003 AA Amster-
} dam, The Netherlands.

\section{Development of the Model}

Consider the intramolecular structure of a surfactant molecule as characterized by the matrix of correlation functions: ${ }^{6}$

$$
\hat{\omega}_{\alpha \gamma}(k)=\left\langle\exp \left[i \mathbf{k} \cdot\left(\mathbf{r}^{(\alpha)}-\mathbf{r}^{(\gamma)}\right)\right]\right\rangle
$$

The pointed brackets indicate equilibrium ensemble average, and $\mathbf{r}^{(\alpha)}$ is the position of atom $\alpha$ of a tagged molecule. The atoms or groups can be partitioned according to whether they are oil-like or water-like-A or B, respectively. The structure functions according to those classifications are

$$
\hat{\omega}_{i j}(k)=\sum_{\alpha \in i} \sum_{\gamma \in j} \hat{\omega}_{\alpha \gamma}(k), \quad i, j=\mathrm{A}, \mathrm{B}
$$

with small wavevector expansion

$$
\hat{\omega}_{i j}(k)=n_{i} n_{j}-k^{2} \Delta_{i j}^{2} / 2 d+\ldots
$$

where $n_{i}$ is the number of atoms of type $i(=\mathrm{A}$ or $\mathrm{B})$ in a surfactant, $d$ is the dimensionality, and

$$
\Delta_{i j}^{2}=\sum_{\alpha \in i} \sum_{\gamma \in j}\left\langle\left|\mathbf{r}^{(\alpha)}-\mathbf{r}^{(\gamma)}\right|^{2}\right\rangle
$$

Asymptotically, the matrix inverse is therefore

$$
\hat{\omega}^{-1}(k) \sim \frac{d}{k^{2} \Delta^{2}}\left[\begin{array}{ll}
1 / n_{\mathrm{A}}{ }^{2} & -1 / n_{\mathrm{A}} n_{\mathrm{B}} \\
-1 / n_{\mathrm{A}} n_{\mathrm{B}} & 1 / n_{\mathrm{B}}{ }^{2}
\end{array}\right] \quad \text { small } k
$$

where $\Delta^{2}=\Delta_{\mathrm{AB}}{ }^{2} / n_{\mathrm{A}} n_{\mathrm{B}}-\Delta_{\mathrm{AA}}{ }^{2} / 2 n_{\mathrm{A}}{ }^{2}-\Delta_{\mathrm{BB}}{ }^{2} / 2 n_{\mathrm{B}}{ }^{2}$ is roughly the

(1) Mittal, K. L., Lindman, B., Eds. Surfactants in Solution; Plenum: New York, 1984.

(2) Connections between surfactant assemblies and the frustration of competing interactions, such as those in the ANNNI model, are found in the lattice formulation of: Widom, B. J. Chem. Phys. 1986, 84, 6943. Aspects of this are explicitly analyzed by: Dawson, K. A. Phys. Rev. A 1987, 36, 3383. Dawson, K. A.; Lipkin, M. D.; Widom, B. J. Chem. Phys. 1988, 88, 5149. A recent review of lattice models for self-assembled systems is given by: Gompper, G.; Schick, M. Lattice theories of microemulsions. In Modern Ideas and Problems in Amphiphilic Science; Gelbart, W. M., Roux, D., Ben-Shaul, A., Eds.; Springer Verlag: New York, 1992. Also see: Hurley, M. M.; Singer, S. J. J. Phys. Chem. 1991, 96, 1938.

(3) Smit, B. Computer Simulation of Phase Coexistence: From Atoms to Surfactants. Ph.D. Thesis, Rijksuniversiteit Utrecht, The Netherlands, 1990.

(4) Smit, B.; Hilbers, P. A. J.; Esselink, K.; Rupert, L. A. M.; van Os, N. M.; Schlijper, A. G. Nature 1990, 348, 624-625. Smit, B.; Hilbers, P. A. J. Esselink, K.; Rupert, L. A. M.; van Os, N. M.; Schlijper, A. G. J. Phys. Chem. 1991, 95, 6361-6368. Smit, B.; Esselink, K.; Hilbers, P. A. J.; van Os, N. M.; Szleifer, I. Preprint, 1991.

(5) Stillinger, F. H. J. Chem. Phys. 1983, 78, 4654-4661.

(6) Chandler, D. Equilibrium theory of polyatomic fluids. In The liquid state of matter: Fluids, simple and complex; Montroll, E. W., Lebowitz, J. L., Eds.; North-Holland Publishing Company: Amsterdam, 1982; p 275-340. 
mean square length of the surfactant molecule.

In the opposite limit

$$
\hat{\omega}_{i j}(k) \sim \delta_{i j} n_{i}, \quad k \rightarrow \infty
$$

Consider now the implications for densities in a volume $V$ :

$$
\rho_{i}(\mathbf{r})=(1 / V) \sum_{\mathbf{k}} \hat{\rho}_{i}(\mathbf{k}) e^{i \mathbf{k} \cdot \mathbf{r}}
$$

At low concentrations $\rho=\left\langle\rho_{\mathrm{A}}(\mathbf{r})\right\rangle / n_{\mathrm{A}}=\left\langle\rho_{\mathrm{B}}(\mathbf{r})\right\rangle / n_{\mathrm{B}}$, small deviations from homogeneity are governed by the Gaussian free energy functional

$$
F_{\mathrm{G}}=\left(k_{\mathrm{B}} T / 2 V\right) \sum_{\mathbf{k}} \sum_{i j=\mathrm{A}, \mathrm{B}} \hat{\rho}_{i}(-\mathbf{k})\left[\hat{\omega}_{i j}^{-1}(k) \rho^{-1}\right] \hat{\rho}_{j}(\mathbf{k})
$$

where $k_{\mathrm{B}} T=\beta^{-1}$ is the temperature times Boltzmann's constant. In particular, with eq 2.8 , it follows from Gaussian statistics (or the principle of equipartition) that the statistical weight, $\exp (-$ $\beta F_{\mathrm{G}}$ ), yields

$$
V^{-1}\left\langle\hat{\rho}_{i}(-\mathbf{k}) \hat{\rho}_{j}(\mathbf{k})\right\rangle=\rho \hat{\omega}_{i j}(k)
$$

which is consistent with eqs 2.2 and 2.7 in the low concentration limit. Equation 2.8 is therefore consistent with the correct second moment at small $\rho$. In view of eqs 2.5 and 2.8 , we see that the free energy cost for fluctuations at small $k$ is prohibitive unless

$$
\lim _{k \rightarrow 0+}\left[\hat{\rho}_{\mathrm{A}}(k) n_{\mathrm{B}}-\hat{\rho}_{\mathrm{B}}(k) n_{\mathrm{A}}\right]=0
$$

Equation 2.10 is the constraint of stoichiometry. The strength of the coupling which enforces this constraint is $k_{\mathrm{B}} T \hat{\omega}_{i j}{ }^{-1}(k)$. Its inverse $k^{2}$ dependence is the same as the Fourier transform of a Coulomb potential. Indeed, precisely the same free energetics govern the small- $k$ Fourier components in a Coulombic model:

$F_{\mathrm{C}}=(1 / 2 V) \sum_{\mathbf{k}} \sum_{i, j=\mathrm{A}, \mathrm{B}} 4 \pi\left[\hat{\rho}_{i}(\mathbf{k}) / n_{i}\right]\left[\hat{\rho}_{j}(-\mathbf{k}) / n_{j}\right] z_{i} z_{j} / k^{2}$

where

$$
z_{\mathrm{A}}=\left(4 \pi \beta \rho \Delta^{2} / d\right)^{-1 / 2}=-z_{\mathrm{B}}
$$

For the Coulombic model, fluctuations that violate charge neutrality are quenched.

Notice that in the Coulombic model, it is natural to group all the A particles within each surfactant and to refer to the density of such groups, $\bar{\rho}_{\mathrm{A}}(\mathbf{r})=\rho_{\mathrm{A}}(\mathbf{r}) / n_{\mathrm{A}}$. Similarly, it is natural to group $\mathrm{B}$ particles referring to $\bar{\rho}_{\mathrm{B}}(\mathbf{r})=\rho_{\mathrm{B}}(\mathbf{r}) / n_{\mathrm{B}}$.

This connection between the constraints of stoichiometry and the constraints of charge neutrality has been noted by Stillinger in a remarkable but generally overlooked paper. ${ }^{5}$ Stillinger identified essentially the same charges $z_{\mathrm{A}}$ and $z_{\mathrm{B}}$, eq 2.12 , and incorporated their interactions into a free energy functional for surfactant-oil-water mixtures. The Coulombic nature of $\hat{\omega}^{-1}(k)$ is found either explicitly or implicitly in earlier work pertaining to pair correlations of interaction site models of molecular fluids. ${ }^{6,7}$ Its utility in thinking about surfactant systems, however, originates with Stillinger.

In the absence of constraints, the statistics of a two-component mixture might be described by free energy functionals of the form

$$
\begin{aligned}
F_{\mathrm{M}}= & \sum_{i=\mathrm{A}, \mathrm{B}} \int \mathrm{d} \mathbf{r} f_{i}\left[\bar{\rho}_{i}(\mathbf{r})\right]-\left(k_{\mathrm{B}} T / 2\right) \times \\
& \sum_{i, j=\mathrm{A}, \mathrm{B}} \int \mathrm{d} \mathbf{r} \int \mathrm{d} \mathbf{r}^{\prime} \bar{\rho}_{i}(\mathbf{r}) c_{i j}\left(\left|\mathbf{r}-\mathbf{r}^{\prime}\right|\right) \bar{\rho}_{j}\left(r^{\prime}\right)
\end{aligned}
$$

where $f_{i}\left[\bar{\rho}_{i}(\mathbf{r})\right]$ is a nonlinear but local free energy density, and $c_{i j}(r)$ is a short-ranged effective interaction (in units of $-k_{\mathrm{B}} T$ ). In the long-wavelength limit, the nonlocal contributions of the latter can be replaced by a square gradient term. A free energy functional $F\left[\bar{\rho}_{\mathrm{A}}(\mathbf{r}), \bar{\rho}_{\mathrm{B}}(\mathbf{r})\right]$ determines the weights in the partition function:

$$
Q=\int D_{\bar{\rho}_{\mathrm{A}}}(\mathbf{r}) \int D_{\tilde{\rho}_{\mathrm{B}}}(\mathbf{r}) \exp \left\{-\beta F\left[\bar{\rho}_{\mathrm{A}}(\mathbf{r}), \bar{\rho}_{\mathrm{B}}(\mathbf{r})\right]\right\}
$$

(7) Chandler, D. J. Chem. Phys. 1977, 67, 1113. Sullivan, D. E.; Gray, C. G. Mol. Phys. 1981, 42, 443. Cummings, P. T.; Stell, G. Mol. Phys. 1982 $46,383$.

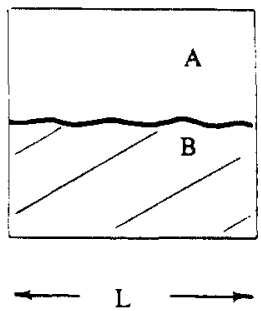

(a)

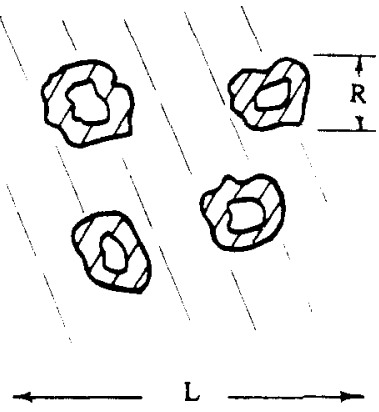

(b)
Figure 1. (a) Phase-separated system of unconstrained A and B particles. (b) Assembled A-B clusters deep within the rich B phase.

With $F=F_{\mathrm{M}}$, it will exhibit phase transitions. For example, a fluid phase rich in component $A$ can separate from one rich in component $B$ provided the temperature is low enough and/or there is sufficient asymmetry among the $c_{i j}(r)$ 's, thereby favoring $\mathrm{A}-\mathrm{A}$ and $B-B$ interactions over those between $A$ and $B$.

Imagine such a phase separation does occur and consider adding to eq 2.13 the free energy $F_{C}\left[\bar{\rho}_{A}^{(s)}(\mathbf{r}), \bar{\rho}_{B}^{(s)}(\mathbf{r})\right]$. Here, $\bar{\rho}_{i}^{(s)}(\mathbf{r})$ refers to the density of a subset of all the particles of type $i$, those which carry the $z_{i}$. The presence of these charges will frustrate the phase-separated system in essentially the same way as the stoichiometric constraints described in the Introduction. Thus, we are led to consider the free energy:

$$
F=F_{\mathrm{M}}\left[\bar{\rho}_{\mathrm{A}}(\mathbf{r}), \bar{\rho}_{\mathrm{B}}(\mathbf{r})\right]+F_{\mathrm{C}}\left[\bar{\rho}_{\AA}^{(s)}(\mathbf{r}), \bar{\rho}_{\mathrm{B}}^{(s)}(\mathbf{r})\right]
$$

where $\bar{\rho}_{i}(\mathbf{r})-\bar{\rho}_{i}^{(s)}(\mathbf{r})$ is the density of the uncharged or free groups of $n_{i}$ particles of type $i$. Stillinger's free energy functional ${ }^{5}$ is of this form. Provided no further detail is required concerning stoichiometric constraints other than its long-wavelength manifestation, this free energy functional should represent a class of models for surfactant assemblies. We explore this possibility in the next two sections of this paper.

\section{Scaling Argument}

Here we use the concept of Stillinger's frustrating charges to develop a scaling theory of self-assembly. The discussion is of an illustrative nature and confined to the simplest possible case.

Assume the conditions are such that the mixture of unconstrained A and B particles phase separates as illustrated in Figure 1a. Far from its critical point, the only appreciable density fluctuations at large length scales are those of the interface with energy $\sim L^{d-1} \sigma$. The parameter $\sigma$ is the surface tension of the A-B interface, $d$ is the dimensionality, and $L^{d}$ is the volume.

To this system, we add symmetric surfactant molecules $A_{n} B_{n}$ at a concentration $\rho$. At large length scales, the effect of this addition is the same as introducing particles possessing the isomorphic frustrating charges:

$$
z_{\mathrm{A}}=-z_{\mathrm{B}}=z \sim\left(1 / \beta \rho \Delta^{2}\right)^{1 / 2}
$$

One such particle with charge $z_{\mathrm{A}}$ is on $\mathcal{A}$ particle representing, in a field theoretic sense, the $n$ A particles of a surfactant molecule. Similarly, each $B$ particle represents $n$ B particles of a surfactant. ( $\mathcal{A}$ and $\mathcal{B}$ refer to fictitious particles used as an aid in visualizing density fields of the $n \mathrm{~A}$ and $n$ B particles per surfactant molecule. $A$ and $B$ are used to indicate particle types and phases.)

Due to the requirement of electroneutrality, these special $\mathcal{A}$ and $\mathscr{B}$ particles must exist together in reasonably close proximity. Therefore, assuming their fraction is more than infinitesimal, both $\mathcal{A}$ and $\mathscr{B}$ charged particles will then exist in, for example, the $B$ phase. Their concentration will be $\rho$. The forced presence of A particles in the B phase frustrates the phase with a free energetic cost of roughly $n \rho L^{d} \epsilon$. Here, $n \epsilon$ is the energy we associate with moving $n \mathrm{~A}$ particles from the $\mathrm{A}$ phase to the $\mathrm{B}$ phase. If the special particles can assemble into large structures, however, the free energetic cost may be lower. 
Imagine self-assembly does occur in the form of micelles as depicted in Figure 1b. In that case, the free energy for placing the isomorphic charged particles in phase $\mathrm{B}$ is

$$
F(R) \sim E_{\text {surface }}+E_{\text {charge }}
$$

where

$$
\begin{gathered}
E_{\text {surface }} \sim N \sigma R^{d-1} \sim \rho L^{d} \Delta^{d} \sigma / R \\
E_{\text {charge }} \sim N R^{d+2}(\delta \rho)^{2} \sim \rho L^{d} \Delta^{d}(\delta \rho)^{2} R^{2}
\end{gathered}
$$

Here

$$
N \sim \frac{\text { (no. of charged particles) } \times \text { (vol of a charged particle) }}{\text { (vol of a micelle) }}
$$

is the number of micelles, $R$ is the typical diameter of a micelle, and $\delta \rho$ is the magnitude of the effective charge density within a micelle. We arrive at (3.2) $-(3.4)$ as follows: Each micelle creates an A-B surface of size $\sim R^{d-1}$ and hence an energy $\sigma R^{d-1}$ where $\sigma$ is the surface tension of the ordinary A-B interface. Further, according to Coulomb's law, the packing of charges within a volume $R^{d}$ leads to the energy $R^{d+2}(\delta \rho)^{2}$ where

$$
\delta \rho \sim z / \Delta^{d}
$$

Here, we are assuming a structure consistent with Figure $1 \mathrm{~b}$ in which one sign change is distributed uniformly in a shell surrounding a nucleus of opposite sign change. The length of a surfactant, $\sim \Delta$, is also roughly the diameters of $\mathcal{A}$ and $\mathcal{B}$ groups. Further, $\Delta \sim n^{l} l$ where $l$ is the diameter of a single A or B particle. For a linear chain, $\nu=1$; for a Gaussian chain, $\nu=1 / 2$.

Notice that $E_{\text {sufface }}$ per cluster resists growth of clusters, in accord with nucleation theory. On the other hand, for a fixed volume, $L^{d}$, this energy favors large clusters over small clusters. This trend competes with that of $E_{\text {charge }}$.

The free energy per unit volume, $F(R) / L^{d}$, is a minimum at $R=R^{*} \sim\left[\sigma /(\delta \rho)^{2}\right]^{1 / 3}$. Comparison of $F\left(R^{*}\right) / L^{d}$ with the counterpart for a nonassembled system, $\sim n \rho \epsilon$, indicates that the assembled system is to be preferred when $n \rho \epsilon \gtrsim \Delta^{d} \rho \sigma^{2 / 3}(\delta \rho)^{2 / 3}$. By making use of eqs 3.1 and 3.5, we therefore predict a crossover in stability at

$$
\rho \sim\left(l^{d-2} / \epsilon^{3}\right) n^{(d-2) \nu-3} \sigma^{2} / \beta
$$

The actual critical micelle concentration will be higher than this density since translational entropy, neglected in this analysis, favors dissociation over assembly. Equations 3.2-3.4 account for intramicelle energetics. Interactions between different micelles are neglected, and entropic effects other than those due to stoichiometry are ignored.

Generalizations of this discussion may be developed for asymmetrical systems and for assemblies more complex than spherical micelles. With the inclusion of translational entropy contributions, such results should be of use in understanding trends such as the molecular size and temperature dependences of critical assembly concentrations and assembly size. The shortcomings of such analyses, however, is that they do not account for fluctuations. We exhibit the nature of these fluctuations in the next section.

\section{Charge Frustrated Ising System}

4.1. Spin Model. The Ising spin system is the simplest model for the physics of phase separation. At each lattice site, representing a microscopic volume, is assigned a spin for which a +1 value indicates the presence of $\mathcal{A}$, and a -1 value indicates the presence of $\mathcal{B}$. Its partition function is

$$
Q=\sum_{\left\{s_{i}\right\}= \pm 1} \exp \left\{\beta J \sum_{i j(n n)} s_{i} s_{j}+(\beta \mu / 2) \sum_{i} s_{i}\right\}
$$

where $\sum_{i j(n n)}$ indicates the sum over nearest-neighbor pairs. This partition function can be cast in the form of (2.14) through the Hubbard-Stratonovich transformation. ${ }^{8}$ For positive $J$ and low

(8) Itzykson, C.; Drouffe, J.-M. Statistical Field Theory; Cambridge University Press: New York, 1989. enough temperatures, the system phase separates with the relative chemical potential, $\mu$, determining which of the two phases, rich in $\mathcal{A}$ or rich in $\mathcal{B}$, is observed. In this section, we modify this standard model by adding frustrating isomorphic charges.

In particular, we consider the Ising model for which a randomly assigned but fixed number of spins carry frustrating charges, $\pm z$. The positive and negative charges are assigned to spin up and spin down particles, respectively. The condition of neutrality coincides with the stoichiometry of surfactants. By fixing the total number of charges (i.e., by employing a canonical ensemble for the surfactant species), we set the value of $z$ in accord with eq 2.12. If we employed an ensemble that allowed the number to fluctuate, the value of $z$ would have to be determined self-consistently in terms of the average surfactant number $L^{d} \rho$.

The interaction energy of the modified Ising system is

$$
-J \sum_{i j(n n)} s_{i} s_{j}+z^{2} \sum_{i>j} s_{i} s_{j} t_{i} t_{j} v\left(r_{i j}\right)
$$

where $r_{i j}$ is the distance between Ising spins $i$ and $j$, and $t_{i}=1$ or 0 depending upon whether or not the Ising spin is charged or not. The function $v(r)$ is the inverse Fourier transform of $4 \pi / k^{2}$ (i.e., $1 / r$ for $d=3$, and $-2 \ln r$ for $d=2$ ). The configurations of this model are sampled by summing over all $s_{i}= \pm 1$ and all $t_{i}=0,1$ such that

$$
M=1 / 2 \sum_{i} t_{i}
$$

is the total number of surfactants, and

$$
0=\sum_{i} t_{i} s_{i}
$$

is the constraint of neutrality.

To simplify this model, we focus specifically on self-assembly, and we consider temperatures well below the critical temperature of the standard Ising model (i.e., the $z=0$ model) and a choice of $\mu$ that places the system well within the spin up phase (i.e., the $B$ phase). In that case, the only pertinent fluctuations are those associated with the frustrating particles-the special spins that carry Stillinger's frustrating charges $\pm z$. A lattice site which is not occupied by a special spin is always a standard Ising up spin. As a result, to within an additive constant and single particle terms, the interaction energy can be reduced to

$U\left(\left(\xi_{i}\right)\right)=-\mathscr{E} \sum_{i j(n n)} \xi_{i} \xi_{j}\left(\xi_{i}-1\right)\left(\xi_{j}-1\right)+z^{2} \sum_{i>j} \xi_{i} \xi_{j} v\left(r_{i j}\right)$

where $\xi_{i}=0,1,-1$ (indicating whether site $i$ contains no special spin, a special up spin, or a special down spin) and $\mathscr{E}=2 J$ expresses the difference between short-ranged "hydrophobic" and "hydrophilic" interactions of the unconstrained $\mathcal{A}$ and $\mathcal{B}$ groups. The energy parameter $\mathscr{E}$ is of the order of $n \in$ used in the scaling argument of section 3. The number of special spins is

$$
2 M=\sum_{i} \xi_{i}^{2}
$$

and the constraint of neutrality or stoichiometry is

$$
0=\sum_{i} \xi_{i}
$$

We have examined the behavior of this model (4.5)-(4.7) by performing a series of Monte Carlo runs for two-dimensional $N$ $\times N$ square lattices. The nearest-neighbor lattice spacing, $\lambda$, corresponds to the length $\Delta$, i.e., the typical spacing between $A$ and $B$ particles within a surfactant. Periodic boundary conditions are employed, and the Ewald sums ${ }^{9}$ associated with

$$
v\left(r_{i j}\right)=\frac{1}{L^{2}} \sum_{\mathrm{k}} \frac{4 \pi}{k^{2}} e^{i \mathbf{k} \cdot r_{i j}}
$$

are performed initially to high precision and stored as an $N(N$ - 1) $/ 2$ array. We have found that Monte Carlo trajectories performed without Ewald sums exhibit artifacts such as layering

(9) Allen, M. P.; Tildesley, D. J. Computer Simulation of Liquids; Clarendon: Oxford, 1987 
commensurate with the minimum image truncation distance. For certain choices of parameters, the charge-frustrated Ising model does exhibit layering, but not of this artificial type.

4.2. Monte Carlo Moves. To obtain reproducible well-equilibrated results, such as layered structures as well as micellar assemblies, it also proves important to utilize various kinds of moves in the Monte Carlo sampling. With conventional one- and two-spin Metropolis moves, the system does assemble quickly, but once an initial structure is formed, no appreciable movement follows.

The particular Monte Carlo moves that we have employed in our simulations are single-spin displacements, "dipole" moves, swapping of positive and negative charges, and cluster moves. Whether a particle move is performed during every Monte Carlo pass depends on the thermodynamic conditions of the system. For example, at low surfactant concentrations, where the frustrating charges are very large in magnitude, the charges of opposite sign are tightly connected to the tails. As a result, the probability of a successful single-spin move becomes very small, and it is important to perform other types of moves.

Single spin moves: In this move, one attempt is made to swap each of the charged spins with one of its eight neighbors. This randomly selected neighbor can be another charged spin or an uncharged spin (solvent site). Note that the solvent fluctuations are ignored, the uncharged spins can simply be regarded as empty sites.

Dipole moves: When the frustrating charges are such that spins with opposite charge are strongly connected, single spin moves - those which result in a separation of these charges-will have a low acceptance ratio. However, when both charges are moved at the same time, the probability of acceptance will be much higher.

In this type of move, which we call dipole move, we first identify a dipole and subsequently displace the two spins. This can be done by randomly selecting a spin, and randomly selecting one of its eight nearest or next-nearest neighbors. If this neighbor has an opposite charge, a dipole is formed; otherwise, the move is rejected. Next an empty site is selected at random; if one of the randomly selected neighboring sites is empty, an attempt is made to move the dipole to these two empty sites. The usual acceptance rules have been used for this type of move. At some conditions, it is favorable to use a form of configurational biased Monte Carlo. ${ }^{10,11}$ This algorithm is described in the Appendix.

Swapping of plus and minus charges: When clusters of charged spins are formed during a simulation, it is important to allow for fluctuations in the polar moment or in the total charge of a cluster. These fluctuation can occur if we swap a plus and minus charge which are in the same cluster or in different clusters respectively.

Cluster moves: Once the charged spins have self-assembled into clusters, it is extremely time consuming to move these clusters via the single-spin moves. Such slow or glassy motion is to be expected for a frustrated system. Our method to circumvent this problem is to utilize collective moves in which we attempt to move an entire cluster. Our algorithm can be viewed as a generalization of that due to Swendsen and Wang, ${ }^{12-14}$ who developed a Monte Carlo procedure for obviating the critical slowing down problem as it occurs in spin systems undergoing phase transformations. The Appendix provides specific details.

4.3. Results of the Simulations. Figure 2 shows typical configurations for a system with $M=125$ at four progressively higher surfactant densities, $\rho \lambda^{2}$. In the progression, the isomorphic charge and the temperature remain fixed with $\beta z^{2} / \lambda=5$ and $\beta \mathscr{E}=5$. The critical temperature of the ordinary two-dimensional Ising model-the $z=0$ system-coincides with $\beta E \approx 1$. Thus, the system is indeed within the $B$ phase. Only the special spins are pictured in the figure. The black circles represent up spins which carry the positive isomorphic charge, and the open circles denote

(10) Siepmann, J. I.; Frenkel, D. Mol. Phys. 1992, 75, 59. press.

(11) Frenkel, D.; Mooij, G. A. M.; Smit, B. J. Phys. Condens. Matter, in

(12) Swendsen, R. H.; Wang, J.-S. Phys. Rev. Lett. 1987, 58, 86-88

(13) Niedermayer, F. Phys. Rev. Lett. 1988, 61, 2026-2029

(14) Li, X.-J.; Sokal, A. D. Phys. Rev. Lett. 1991, 67, 1482-1485

(15) Edwards, R. G.; Sokal, A. D. Phys. Rev. A 1988, 38, 2009-2012. (a) $\bar{\rho}=0.05$

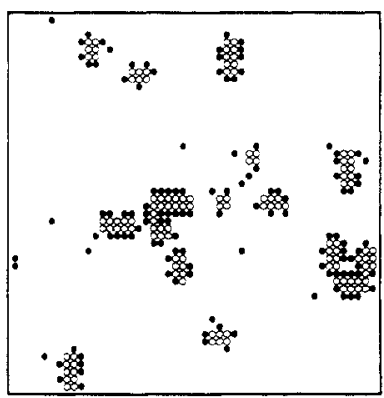

(c) $\bar{\rho}=0.2$

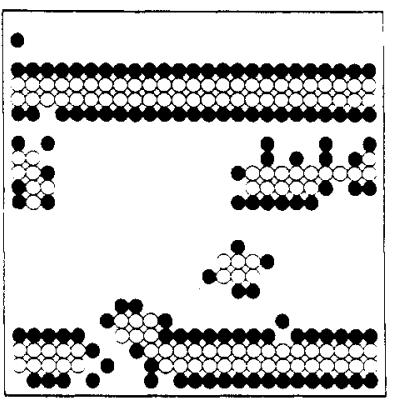

Figure 2. Snapshots of some typical configurations of the charge frustrated Ising system. The open spheres (0) represent the $\mathcal{A}(-)$ spin;

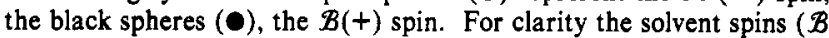
spins without a charge) are not drawn. The number of charged $\mathcal{A}, \mathcal{B}$ spins is 125 , the isomorphic charge $z=2.24(\lambda / \beta)^{1 / 2}$, and hydrophobic-hydrophilic interaction $\mathscr{E}=5 / \beta$. In (a) the length of the box is $L$ $=50 \lambda$ and the corresponding density is $\rho=0.05 / \lambda^{2}$; in (b), $L=35 \lambda$ and $\rho=0.10 / \lambda^{2}$; in (c), $L=25 \lambda$ and $\rho=0.20 / \lambda^{2}$; and in (d), $L=20 \lambda$ and $\rho=0.31 / \lambda^{2}$.

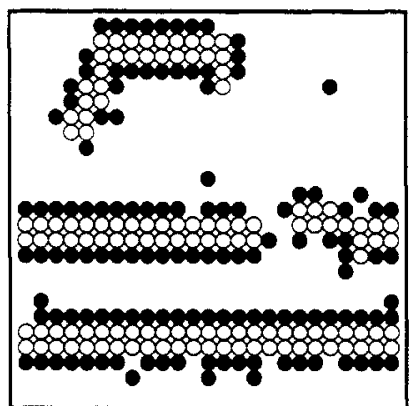

(a)

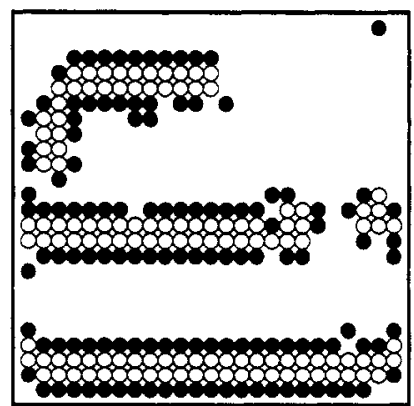

(b)

Figure 3. Two snapshots of the system separated by 200 Monte Carlo passes. The system is identical to the one of Figure $2 \mathrm{c}$.

the down spins which carry the negative isomorphic charge. Roughly speaking, therefore, regard the black and white particles as the head and tail groups in a surfactant assembly. This perspective is not literally true, however, since these pictures depict a field theoretical model. Averages of the spatial patterns observed in these pictures do coincide with the average behavior of the head and tail density fields. As such, the observer should not be dis- 
(a)

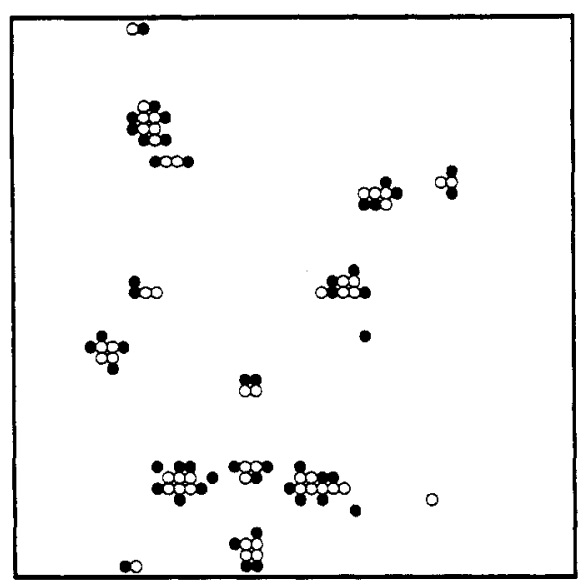

(c)

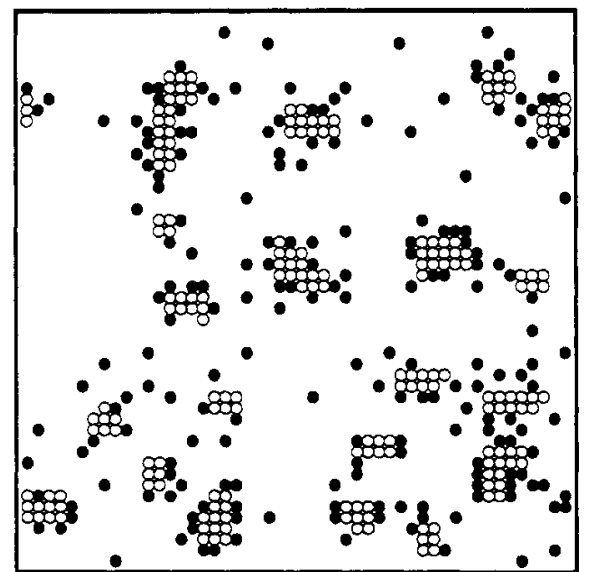

(b)

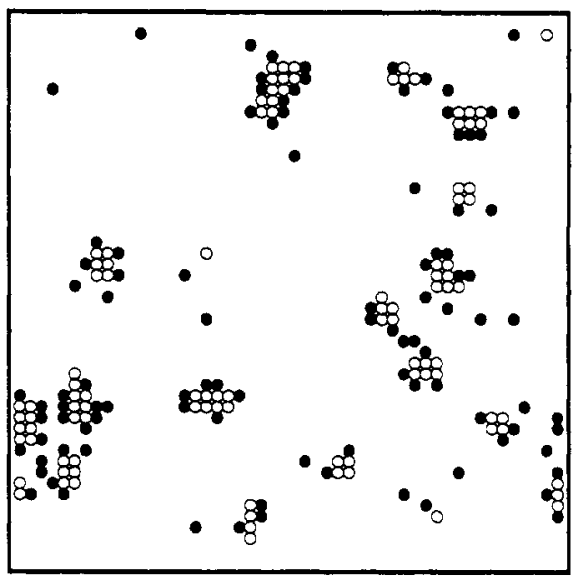

(d)

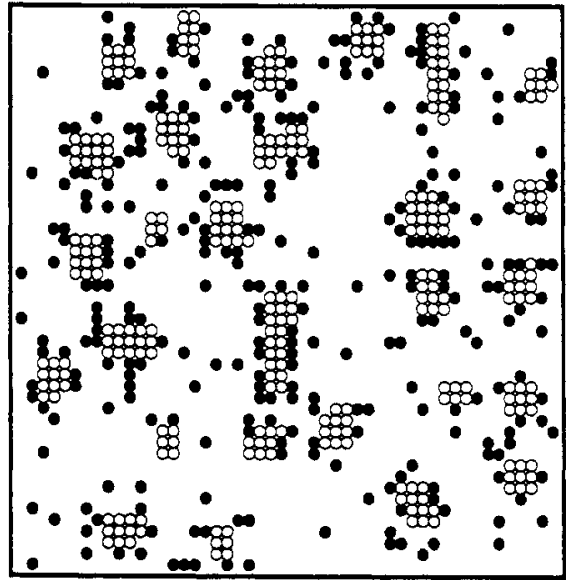

Figure 4. Snapshots of some typical configurations of the charge-frustrated Ising model for various surfactant concentrations. The temperature corresponds to $\beta E=3.75$, the isomorphic charge is $z=(0.088 \lambda / \beta \bar{\rho})^{1 / 2}$, and the length of the simulation box is $L=50 \lambda$. The concentration of charged $\mathcal{A}, \mathcal{B}$ particles is in (a) $\rho=0.02 / \lambda^{2}$, in (b) $\rho=0.04 / \lambda^{2}$, in (c) $\rho=0.08 / \lambda^{2}$, and in (d) $\rho=0.12 / \lambda^{2}$.
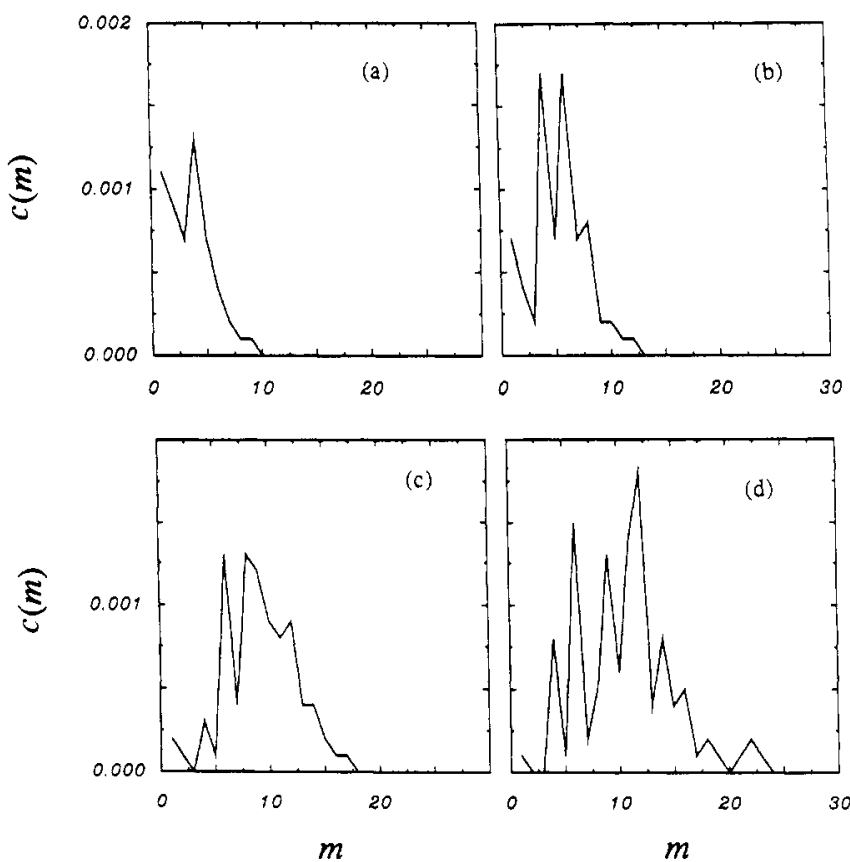

Figure 5. Concentration, $c(m)$, of clusters containing $m \mathcal{A}$ particles (i.e., $m$ charged negative spins). The thermodynamic conditions of panels $(a-c)$ are as those in Figure 4. These concentrations were obtained by averaging over at least 10000 Monte Carlo passes.

tressed to see small length scale fluctuations that would seem to violate the stoichiometric connectivity of surfactant particles (as opposed to fields).
The Monte Carlo trajectories have been carried out to sufficient length to convince us that the exhibited patterns are indeed representative of equilibrium in these finite systems. As evidence we show in Figure 3 two additional configurations at the reduced density $\bar{\rho}=\rho \lambda^{2}=0.2$. The two configurations in Figure 3 are displaced by 200 passes. In all cases, we equilibrate the system for at least 10000 passes.

The progression in Figure 2 begins at a low density where simple micellar assemblies are apparent. It is evidently above the critical micelle concentration. As density increases, the structures of these assemblies elongate, and at reduced density $\bar{\rho}=0.4$, one observes bilayers in equilibrium with micelles. At yet higher densities, these bilayers form what appears to be a lamellar structure (with defects). The simulated system, however, is relatively small. Due to long-wavelength fluctuations that must exist in this two-dimensional system, it may be that the orientation of these aligned patterns does not persist to long length scales.

Figure 4 shows representative configurations at a temperature $\beta \mathscr{E}=3.75$ and densities ranging from $\bar{\rho}=0.02$ to $\bar{\rho}=0.12$. The isomorphic charge is decreased with increasing $\rho$ so as to coincide with an isothermal compression with $\beta z^{2} / \lambda=0.088 / \bar{\rho}$ (see eq 3.1). The cluster size distributions corresponding to these configurations are shown in Figure 5. The distribution of Figure $5 \mathrm{~b}-\mathrm{d}$ exhibits the signature of micellar assembly. The irregular shape of the distribution curves is caused by the underlying lattice. In a continuum version of the model, the distributions would be more regular. Figures $4 \mathrm{a}$ and $5 \mathrm{a}$ show the results for $\bar{p}=0.02$, which is below the critical micelle concentration.

At very low concentrations, the strength of the frustrating charge becomes very large. In this regime on a square lattice, we find that head-tail dipoles bind as dipolar pairs to form a rarified gas of quadrupoles (an indication of this dipole pairing can be seen in Figures $4 a$ and $5 a$ ). The binding-unbinding 
transition which is present in this system at extremely low $\rho$ occurs in regions of parameter space that do not seem relevant to the phenomena of large length scale assembly.

The spontaneously assembled structures observed in Figures 2-4 are much like those found in nature. They are remarkable in light of the underlying model's simplicity. Accounting for stoichiometric constraints at only the longest length scales frustrates the normal Ising system with competing Coulombic interactions. These interactions alone are sufficient to produce the observed self assemblies.

Due to screening, the charge-charge correlations need not be long ranged. In the random phase approximation or DebyeHückel theory, the inverse screening length is $\left(1 / \beta \rho z^{2}\right)^{1 / 2} \sim \Delta$, i.e., the size of the surfactant molecule or the nearest-neighbor spacing of the lattice model. The Debye-Hückel estimate is not accurate for the highly correlated systems we have simulated Further, correlations along surfaces will be long ranged. Nevertheless, it would appear from Figures 2-5 that the actual screening lengths (normal to surfaces) are relatively short.

By adding real charges along with the frustrating charges, the picture we have drawn herein can be broadened to include ionic surfactants. At extremely low ionic strength, the resulting long screening length may qualitatively alter the behaviors of the system we have thus far examined. At moderate to high ionic strength, however, the solvent averaged interactions between surfactants will be reasonably short ranged. In that case it may be that the primary effects of ionic interactions is to make the energy parameter, $\mathscr{E}$, a function of ionic strength.

\section{Discussion}

In this paper, we have developed the concept of frustrating charges. We have shown that this concept provides a convenient framework for making qualitative estimates pertaining to self assembly. Further, we have used the concept to motivate a new model - the charge frustrated Ising system-and we have carried out simulations of the model by employing a novel procedure of cluster moves. It is apparent that the charge-frustrated Ising system exhibits rich behavior. It remains to be seen how many features of self-assembly can be understood accounting for only the longest wavelength aspects of these systems. Further study of the charge-frustrated Ising model in both two and three dimensions will be helpful in this regard. Ultimately, however, we believe the most important virtue of the perspective we call attention to herein is the possibility that it will provide a convenient basis for off-lattice theories of self-assembling fluid systems.

Concerning the possible scaling theories, the argument employed in section 3 might be extended to treat block polymers. That case, however, will probably require a somewhat more complete accounting of entropic effects than the one we have illustrated. An extended treatment of entropic effects may also become pertinent if one attempts to use these arguments to treat assemblies more complex than spherical micelles.

In view of the Monte Carlo results of section 4, however, it is perhaps wise to remain skeptical with regard to predictions derived from these mean-field arguments. The Monte Carlo simulations exhibit a high degree of polydispersity and variation in cluster shapes. A complete theory should account for these fluctuations. We hope that charge-frustrated models will provide a simple enough framework that this accounting can be made.

Acknowledgment. We are grateful to Kenneth Dawson for stimulating the research reported herein. In addition, we have benefited from discussions with Michael Deem, Matthew Fisher, and Lawrence Pratt. This research has been supported by $\mathrm{Na}$ tional Institutes of Health and by the National Science Foundation. A semester's leave from academic responsibilities other than research was generously provided to D.C. by the Miller Foundation.

\section{Appendix: Dipole and Cluster Moves}

In this Appendix we describe the algorithms that we have used for the dipole moves and cluster moves.

Dipole Moves. The algorithm we have used consists of the following steps:

(1) An $\mathcal{A}$ or $\mathscr{B}$ spin is selected at random. The position of this root spin is called $i_{1}$.
(2) The number of neighbors of opposite sign of the root spin is determined. This number is called $n_{1}$. We consider the eight nearest and next-nearest neighbors. If $n_{1}=0$, the move is rejected; otherwise one of these $n_{1}$ spins is selected at random. The location of this site is called $j_{1}$. This spin together with the root spin defines the dipole which will be attempted to be moved. Furthermore, the number of empty (solvent) sites among the eight neighbors is determined, this number +1 is called $n_{1}^{e}$.

(3) The energies of the spins at sites $i_{1}$ and $j_{1}, u\left(i_{1}\right)$ and $u\left(j_{1}\right)$, respectively, are calculated. In these energies we exclude the interactions between the two spins of the dipole.

(4) An empty site, $i_{2}$, is selected at random, and with equal probability the $\mathcal{A}$ or $\mathscr{B}$ side of the dipole is placed at this position. The energy of this spin is $u\left(i_{2}\right)$.

(5) The number of empty neighboring sites of $i_{2}$ is determined. This number is called $n_{2}^{e}$. If $n_{2}^{e}=0$, the move is rejected; otherwise, one of these sites is selected at random. This site is $j_{2}$ and the energy of the spin placed at this position $u\left(j_{2}\right)$. Note that in the energies $u\left(i_{2}\right)$ and $u\left(j_{2}\right)$ the interactions between the spins of the dipole are again excluded. In addition, the number of spins with of opposite charge of the spin placed at position $i_{2}$ is calculated. This number +1 is $n_{2}$.

(6) This dipole move is accepted with a probability given by

$$
\operatorname{acc}(1 \mid 2)=\min \left(1, \frac{W_{2}}{W_{1}}\right)
$$

where

$$
\begin{aligned}
& W_{1}=n_{2} n_{1}^{e} \exp \left\{-\beta\left[u\left(i_{1}\right)+u\left(j_{1}\right)\right]\right\} \\
& W_{2}=n_{1} n_{2}^{e} \exp \left\{-\beta\left[u\left(i_{2}\right)+u\left(j_{2}\right)\right]\right\}
\end{aligned}
$$

We will now show that this scheme indeed samples according to a Boltzmann distribution of states. To do this we follow the development of refs 10 and 11 imposing detailed balance:

$$
P(1 \mid 2) \operatorname{acc}(1 \mid 2) \Gamma(1)=P(2 \mid 1) \operatorname{acc}(2 \mid 1) \Gamma(2)
$$

in which $\Gamma$ is the density of states, $P(1 \mid 2)$ is the probability of generating configuration 2 out of 1 , and $a c c$ is the acceptance probability. According to the algorithm above

$$
P(1 \mid 2)=\frac{1}{n_{1}} \frac{1}{n_{2}^{e}}
$$

in which we have ignored factors which are the same for every move. A similar expression can be written for $P(2 \mid 1)$ :

$$
P(2 \mid 1)=\frac{1}{n_{2}} \frac{1}{n_{1}^{e}}
$$

Substituting of (A.5) and (A.6) into (A.4) and assuming that $W_{2}<W_{1}$ and that we sample a Boltzmann distribution, we obtain

$$
\frac{1}{n_{1}} \frac{1}{n_{2}^{e}} \frac{W_{2}}{W_{1}} \exp [-\beta U(1)]=\frac{1}{n_{2}} \frac{1}{n_{1}^{e}} \exp [-\beta U(2)]
$$

in which $U(i)$ is the total energy of configuration $i$. Substitution of (A.2) and (A.3) gives

$$
\begin{aligned}
& \exp \left\{-\beta\left[u\left(j_{2}\right)+u\left(i_{2}\right)\right]\right\} \exp [-\beta U(1)]= \\
& \exp \left\{-\beta\left[u\left(j_{1}\right)+u\left(i_{1}\right)\right]\right\} \exp [-\beta U(2)]
\end{aligned}
$$

which proves that detailed balance is obeyed since

$$
U(1)-U(2)=\left[u\left(j_{1}\right)+u\left(i_{1}\right)\right]-\left[u\left(j_{2}\right)+u\left(i_{2}\right)\right]
$$

These dipole moves can also be achieved if we replace step 2 of the algorithm by simply a random selection of one orientation and if this is not a particle of opposite sign the move will be rejected. The same procedure can be used in step 4 . When such a scheme is used, the ordinary acceptance rules apply. On the basis of simple estimates, one may argue that the probability of moving a dipole in the biased scheme, depending on the densities and assembled structures, is $\mathbf{8 - 3 2}$ higher than in the ordinary Monte Carlo scheme. At some conditions, the increase justifies the extra calculations necessary for the biased scheme.

Cluster Moves. Over the last few years, significant progress has been made in the development of algorithms which are efficient for simulations of large lattice systems near criticality. ${ }^{12-15}$ At these conditions the standard, single-spin-flip Monte Carlo moves suffer from severe critical slowing down. These new algorithms reduce this critical slowing down significantly. ${ }^{14}$ The idea is to 
group lattice sites which have the same spin into clusters and to generate a new configuration by flipping all spins on a cluster in one Monte Carlo step. As a result one can obtain large configuration changes. To obtain such a change using the standard Monte Carlo scheme would require many sweeps through the lattice. In this Appendix, we generalize the approach of Swendsen and Wang to permit the movement of clusters. These generalized cluster moves can be used for lattice models and continuum models. Although we refer to spins on a lattice, the same equations hold for particles moving in continuum space. For clarity we consider a pure component system consisting of empty sites and spins (lattice gas); the generalization to include spins of different kinds is straightforward.

Following Niedemayer, ${ }^{13}$ we assume that we connect two spins, labeled $l$, via a bond with a probability $p$

$$
p=p\left(r_{l}\right)
$$

where $r_{l}$ is the distance between a pair of spins, and $p(r)$ is a function which can be chosen arbitrarily, provided that $0<p(r)$ $<1$.

A cluster is defined as the set of spins which are connected to each other via a path of bonds. Note that the smallest cluster is a single spin. The probability of obtaining a configuration of clusters $C$ from a configuration $S$ of spins is given by

$$
P[C(S)]=\sum_{B(C)} \prod_{l \in B} p\left(r_{l}\right) \prod_{l \in B}\left[1-p\left(r_{l}\right)\right]
$$

where the summation runs over all different bond configurations which give the same cluster configuration $C$. The first product is over all pairs of spins which form a bond, and the second product is over all spins which do not form a bond. Now we change the cluster configuration $C$ to obtain the new cluster configuration $C^{\prime}$. From this cluster configuration we can obtain a new configuration of the spins, $S^{\prime}$, by removing the bonds. Important is that the configuration of spins within a cluster has not been affected by this move.

The acceptance rules for these moves can be derived if we impose detailed balance, i.e.

$\Gamma(S) P[C(S)] \operatorname{acc}\left[C(S) \mid C^{\prime}\right]=\Gamma\left(S^{\prime}\right) P\left[C\left(S^{\prime}\right)\right] \operatorname{acc}\left[C^{\prime}\left(S^{\prime}\right) \mid C\right]$

in which $\Gamma(S)$ is the density of states $S$. Detailed balance is obeyed if the acceptance rules satisfy (A.12). Detailed balance is certainly guaranteed if we demand that detailed balance is obeyed for any particular choice of bonding configuration, or

$$
\begin{aligned}
& \exp [-\beta U(S)] \prod_{l \in B} p\left(r_{l}\right) \prod_{l \in B}\left[1-p\left(r_{l}\right)\right] \operatorname{acc}\left[C(S) \mid C^{\prime}\right]= \\
& \quad \exp \left[-\beta U\left(S^{\prime}\right)\right] \prod_{l \in B} p\left(r_{l}^{\prime}\right) \prod_{l \in B}\left[1-p\left(r^{\prime}\right)\right] a c c\left[C^{\prime}(S) \mid C\right]
\end{aligned}
$$

in which we have assumed that we sample a Boltzmann distribution of states.

We can write the energy $U$ as the sum of contributions from interactions between spins which belong to different clusters and the interactions between pairs residing on the same cluster. Since the configurations of spins within a cluster have not changed while going from cluster configuration $C$ to $C^{\prime}$, we have for the intramolecular cluster energy

$$
U^{\text {intraclus }}(S)=U^{\text {intraclus }}(S)
$$

Similarly, we can write the probability of forming a cluster configuration as the product of forming bonds between spins which are in the same cluster and which are on different clusters. Since, the probability of forming bonds within a cluster has not changed while going from cluster configuration $C$ to $C^{\prime}$, we can write

$$
P^{\text {intraclus }}[C(S)]=P^{\text {intraclus }}\left[C^{\prime}(S)\right]
$$

Using (A.14) and (A.15), we can reduce (A.13) to

$$
\begin{aligned}
\operatorname{acc}\left\{C(S) \mid C^{\prime}\right] & \prod_{l \in B^{*}}\left\{\left[1-p\left(r_{l}\right)\right] \exp \left[-\beta u\left(r_{l}\right)\right]\right\}= \\
\operatorname{acc}\left[C^{\prime}\left(S^{\prime}\right) \mid C\right] & \prod_{l \in B^{*}}\left\{\left[1-p\left(r_{l}^{\prime}\right)\right] \exp \left[-\beta u\left(r_{l}^{\prime}\right)\right]\right\}
\end{aligned}
$$

where $B^{*}$ denotes that in the product only those pairs of spins are considered which belong to different clusters. Furthermore we have used

$$
U^{\text {interclus }}(S)=\sum_{l \in B^{*}} u\left(r_{i}\right)
$$

Detailed balance is therefore obeyed if we choose for the acceptance rules

$\frac{a c c\left[C(S) \mid C^{\prime}\right]}{\operatorname{acc}\left[C^{\prime}\left(S^{\prime}\right) \mid C\right]}=\frac{\prod_{l \in B^{*}}\left\{\left[1-p\left(r_{l}\right)\right] \exp \left[-\beta u\left(r_{l}\right)\right]\right\}}{\prod_{l \in B^{*}}\left\{\left[1-p\left(r^{\prime}\right)\right] \exp \left[-\beta u\left(r_{l}^{\prime}\right)\right]\right\}}$

There are many choices that satisfy equation (A.18), an obvious choice is the Metropolis form

$\operatorname{acc}\left[C(S) \mid C^{\prime}\right]=\min \left(1, \frac{\prod_{l \in B^{*}}\left[1-p\left(r_{l}\right)\right] \exp \left[-\beta u\left(r_{l}\right)\right]}{\prod_{l \in B^{*}}\left[1-p\left(r_{l}^{\prime}\right)\right] \exp \left[-\beta u\left(r_{l}^{\prime}\right)\right]}\right)$

It is instructive to consider (A.18) in more detail. First note that we can recover the ordinary Monte Carlo scheme if we set $p(r)=0$, i.e., the probability of forming a bond is zero. (A.18) shows that we can move clusters instead of spins in a Monte Carlo procedure and still sample the Boltzmann distribution of the spins provided that we correct, via the acceptance rule, for the bias introduced by the artificial bonds.

Consider, for example, the bond function

$$
p(r)=\left\{\begin{array}{lll}
1 & \text { if } & r<d \\
0 & \text { if } & r>d
\end{array}\right.
$$

If we use this bonding function, spins will be grouped if the distance between two spins is smaller than $d$. (A.18) states that we must reject all moves that bring two spins of different clusters at a distance smaller than $d$. These moves must be rejected. For if two, initially separated, clusters move in such a way that they will have a bond between them in the next move, they will in this next move be considered as one cluster. It will then be impossible to separate them to retrieve the initial configuration. Such a move would therefore violate detailed balance and is thus rejected.

In our simulations we have used the following version of the cluster moves:

(1) An $\mathcal{A}$ spin is chosen at random, and the cluster to which this charged spin belongs is determined. The site of this spin is $i$. As candidates for forming a bond, we consider only the four nearest neighbors; for distances further away the probability of forming a bond is 0 . For these neighbors, we use as a bonding function

$$
p\left(\xi_{i} \xi_{j}\right)=\left|\xi_{i} \xi_{j}\right|
$$

which makes a bond between the particles $i$ and $j$ if both particles are charged spins.

(2) An attempt is made to displace the entire cluster to one of the eight neighboring positions.

(3) If the cluster is moved to one of the four next nearest neighbors, the move is rejected if it cause an overlap with another cluster.

(4) This move is accepted with a probability (see (A.19))

$$
\operatorname{acc}(\text { old } \mid \text { new })=\min \left(1, \frac{\prod_{\text {the edge }}\left[1-p\left(\xi_{i} \xi_{j}\right)\right] \exp [-\beta U(\text { new })]}{\exp [-\beta U(\text { old })]}\right)
$$

where $U$ (new) is the energy of the new configuration. This equation follows directly from (A.19), if we recall that by construction the edge of the cluster in the old position does not contain any other charged spins and since we displace the cluster over only one lattice site so that in the new configuration the only possible contact with other clusters, which are in this case single charged spins, is at the edge of the cluster. From this equation it follows that a move will be rejected if at the edge of the cluster another charged spin is located. Note that the edge is defined as the nearest neighbors of the spin at the border of the cluster, excluding those sites which are already occupied by spins belonging to the same cluster.

Of course, several different choices of bonding functions and cluster moves can be made. 